\title{
3D environmentally friendly concrete printing model preparation
}

\author{
Yuriy Lazarev ${ }^{1}$, Oleg Krotov ${ }^{1, *}$, Svetlana Belyaeva ${ }^{1}$, and Marina Petrochenko ${ }^{1}$ \\ ${ }^{1}$ Peter the Great St.Petersburg Polytechnic University, Polytechnicheskaya Str.29, 195251, \\ St.Petersburg, Russia
}

\begin{abstract}
This article considers ways of the construction printing of models for agriculture, road, civil and also industrial construction using concrete mixtures. For acquaintance with technology, the architectural element with width of layer of $4 \mathrm{~cm}$ and $8 \mathrm{~cm}$ all model high has been taken with height of one layer of $2 \mathrm{~cm}$. This model has been prepared with use of two packages of the program complexes having different functionality, namely AutoCAD+SheetCAM+Mach3, the second - Sketch-Up+Simplify3D. Each software package was used for design of model in 2D or 3D perspectives, division of model into layers, identical on height, by means of technology of slicer, and also for creation of task of the model printing by concrete for the construction printer of model S 6044. Ready mixes for geopolymer concrete have been taken. By results of the printing, comparison of quality of the models printed on the construction printer and technology of each package of program complexes have been made. The printing of models has shown that quality of the printing is identical. In this case, the second method using a bundle of 2 programs (SketchUp + Simplify3D), which allows printing volumetric models of any shape both in plan and in the future, has an advantage.
\end{abstract}

\section{Introduction}

The 3D printing of concrete (3DCP) is the perspective direction of research which main objective consists in automation of construction processes, by means of such advantages as: continuous work, economy in money and human resources, high quality of the final products. [1-5] This type of the printing consists in extruding of concrete mix from printer nozzle on the trajectory set in computer programs [6-9].

The software plays important role in work with the construction 3D printing, but now there is no integrated program for full cycle of the printing that creates some difficulties, in particular in increase in time of preparation of model, using several program complexes [1012].

Before printing any 3D object by means of the construction printer, it is necessary to perform three obligatory operations:

1. Creation of trajectory of the movement of printing head of the printer;

2. Preparation of the received route by means of the program - slicer whose purpose consists

* Corresponding author: krotov.om@edu.spbstu.ru 
in division of model into parts, equal on height, - printing layers;

3. Loading of object in program complex - the operator who is responsible for operation of the printer during the printing namely for installation of certain speed of the printing, rotation of the screw.

For performance of above-mentioned operations of the company in the field of the additive technologies in construction use 2 approaches in the printing of concrete objects:

1. The printing concrete on the basis of CAD drawings;

2. The printing concrete on the basis of volume models of the OBJ, STL, IFC formats and others.

The first approach is followed by the Russian company of the Spetsavia specializing in development of construction printers. This company uses the following set of programs: $\mathrm{CAD}+\mathrm{SheetCAM}+\mathrm{Mach} 3$ [13]. This way is characterized by simplicity of the printed objects as the $2 \mathrm{D}$ drawing is taken as basis of future three-dimensional object, that is in given cases the printing of objects with apertures and inclined elements is at a loss. To print difficult elements in such a way, it is necessary to edit the text file with the unique code of object intended for work with the 3D printer (G-code) manually. Nevertheless, the Spetsavia has managed to print the house, using this method.

The second way of the printing concrete the most popular as allows to realize one of the main objectives of the construction printing - creation of curvilinear forms in the plan and perspective. The CONprint3D company in article devoted to the construction printing considers use at the heart of the construction printing 3D objects of the STL, OBJ, IFC, STEP formats and others [14]. Such way allows the designer to prepare house model in such programs as Revit, ArhciCAD, and to further use this model in the program allowing to make operation of the printing, however, not all programs correctly work with the construction printer as are intended for the printing of plastic.

Both ways of the printing have at the same time both advantages, and shortcomings, but the question of replacement of one way completely remains to others unresolved.

The purpose of work consists in comparison of results of the printing of identical element on the construction printer created by means of two software packages, namely AutoCAD+SheetCAM+Mach3, the second - SketchUp+Simplify3D.

For achievement of this purpose it is necessary to do the following tasks:

1. To choose element which will be printed;

2. To prepare this element for the printing by means of two packages of program complexes;

3. To make the printing of elements on the construction printer.

\section{Methods}

\subsection{Materials}

For conducting experiment ready-made compounds from which geopolymer concrete is subsequently obtained have been taken. This material replaces Portland cement in the construction printing today, allowing to reduce emissions of $\mathrm{CO} 2$ in the atmosphere. Composition of mix of geopolymer concrete for one layer of the printing is specified below: fine aggregate - quartz sand $(2,871 \mathrm{~kg})$, geosilicate $(0,754 \mathrm{~kg})$, geopolymer cement $(2,175$ $\mathrm{kg})$.

\subsection{Programs}

With wide choice of program complexes the following programs have been chosen as the 
main in the first software package for preparation and the construction printing of model:

- AutodeskAutoCAD (program for design of model)

- SheetCam (slicer);

- Mach3 (operator of the concrete printer).

In the AutodeskAutoCAD program when using versatile tool - spline - it is possible to create elements of any form and bend, also these models can be adapted further for movement them in other programs. By preparation of model the internal scale 1:1 as programs of the following stage are oriented only to it [15-19] is strictly used. After drawing of sketches of object of the site, it is necessary to make its preservation in the format demanded for the program - slicer, but it is preferable in the standard format "* dxf". The program has no apparent defects.

Slicer - SheetCam is developed for creation of G-code (the programming language managed the numerical device of the software earlier) for CNC [20-21] machines. After import of the drawing from the AutoCAD program it is necessary to connect the image of model with model in SheetCam by definition of its such characteristics as the model height, number and thickness of layers, speeds of trajectory and rotational speed of the screw. After preparation of model the program provides code $\mathrm{G}$ in format for the printing. * tap. The main lack of this program consists in weak adaptation to certain operation of the construction printer. The main problem is the programmed continuous concreting. When the model consists of repeated contours, the extruder continues to produce mix during the movement to basic point, thus destroying original form of model and destroying its set structure. It also leads to the big consumption of construction materials influencing economic component of construction process.

The code G created in SheetCam is loaded into Mach3 - the program - the operator of the construction printing where manage the printer. The program defines any position of initial coordinates of model at the level of site which is convenient during the work with large number of models which have to be located in strictly certain section to working site; it is also possible to correct the speed of extruder and rotation of the screw directly during the printing. In special cases, the speed of extruder cannot be changed that badly influences quality of the printing. This problem consists in the programmable code $\mathrm{G}$ which is keeping one speed of constant and not allowing it to be changed. Set of three programs allows to print interesting curve forms, but it is impossible to print the forms having inclination in the vertical plane and also with the different changing forms on height, for example, apertures, pyramids, domes. Thus, it is impossible to print volume model. To make it, it is necessary to change manually the code $\mathrm{G}$ created in SheetCam or to generate several codes for different models that is more laborious work. It is important to note that the only combination of the printing of repeated models increases risk of change of basic coordinate that can result in the wrong result. By consideration of this work the model printing in the form of chair has been studied. Printing Model height $-8 \mathrm{~cm}$.

Originally, AutoCAD does not determine the object height because the model submits the flat drawing. All curve forms have been made earlier mentioned tool - spline. After creation $\mathrm{G}$ code, it is necessary to give in it the command for stop of rotation of the screw at the movement of printing head to basic point as in the absence of stop of rotation of the screw mix continues to come out nozzle that leads to violation of integrity of the printed object. There is no automatic function directly in SheetCam, thus, it is necessary to program manually stop of rotation of the screw for each layer in G-code. 


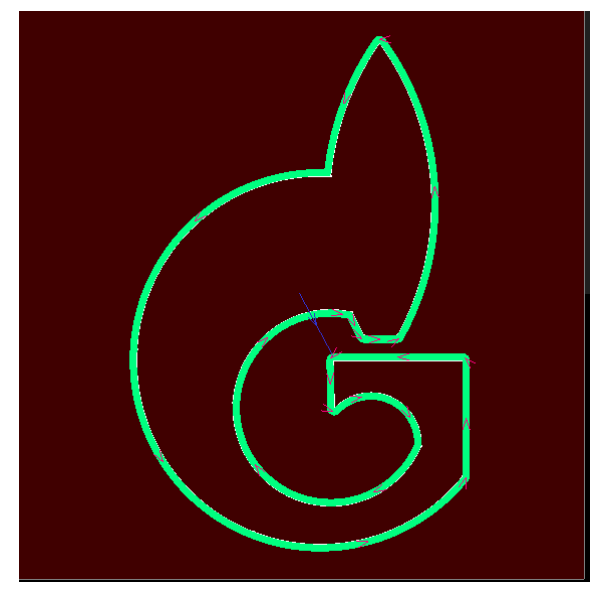

Fig. 1. Model in SheetCam.

In Mach3, during the printing, it is necessary to correct the extruder speed, and also rotating speed of the screw as these actions determine contour thickness, and also its uniformity. As the printer works by the principle of continuous concreting through nozzle, at the long printing mix can begin to grab in the capacity of printing head that leads to stop of process of the printing, clearing of printing head and increase in time of the printing of model. In most cases at violation of process of the printing the printed element will have the broken structure when resuming the printing from top ply.

Simplify3D provides functions and slicer, and the machine controller preventing creation of additional formats. Before to import model to Simplify3d, this object has been prepared in the program for design of volume model, such as SketchUp.

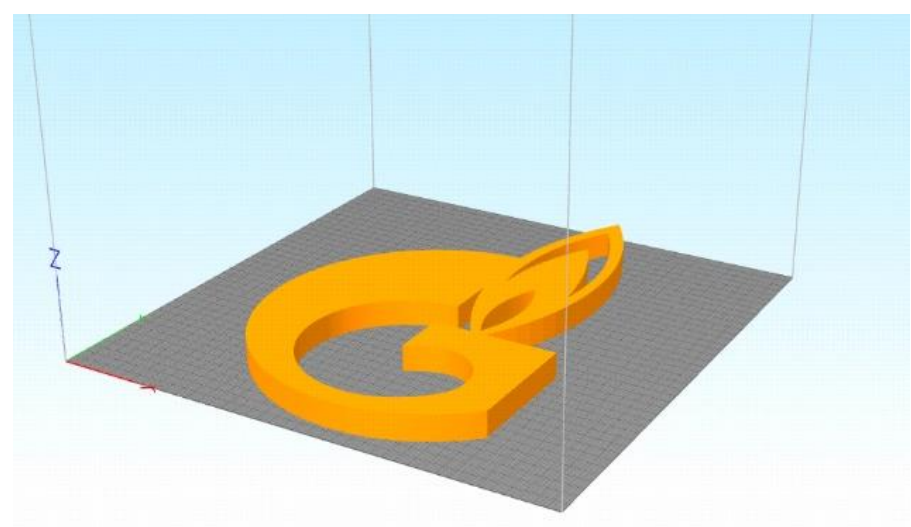

Fig. 2. Model in Simplify3d.

Thickness of element has been chosen as $4 \mathrm{~cm}$, height $-8 \mathrm{~cm}$. The file of model has been saved in the OBJ format and then is open in Simplify3d. There was no need to set the direction of the printing in Sipmlify3d. In the program it has been made automatically.

Both ways of model preparation are shown in the figure 3. 


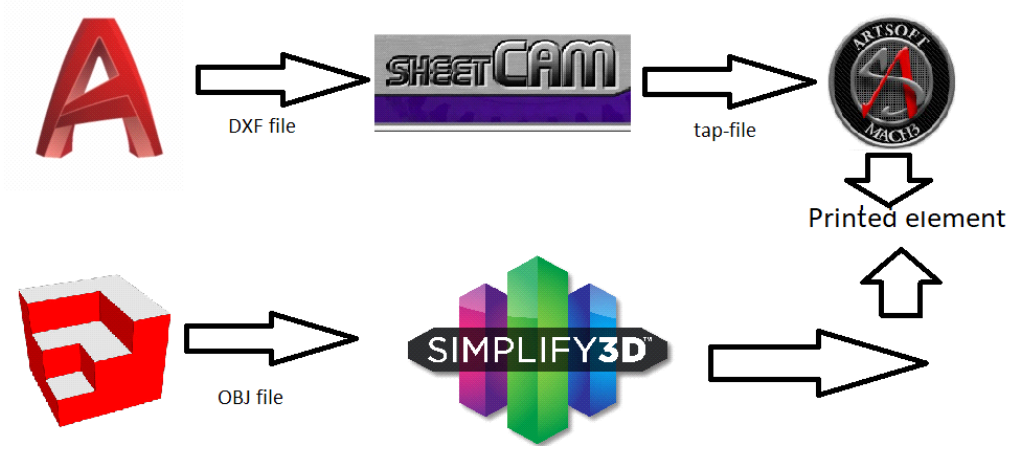

Fig. 3. Preparation of printing models.

\section{Results and Discussion}

To distinguish printed models, different color pigments have been added to both mixtures. Both printed models have the identical curve forms that means the same trajectories of enrollment in SheetCam and Simplify3d.

Also, there were no indistinct layers in both elements because of opportunities of Mach3 and Simplify3d to regulate the speed of specific extrusion. All programs equally had high efficiency during the work with the specific printer. Speed of extrusion has been chosen as 4 $\mathrm{cm} / \mathrm{sec}$.

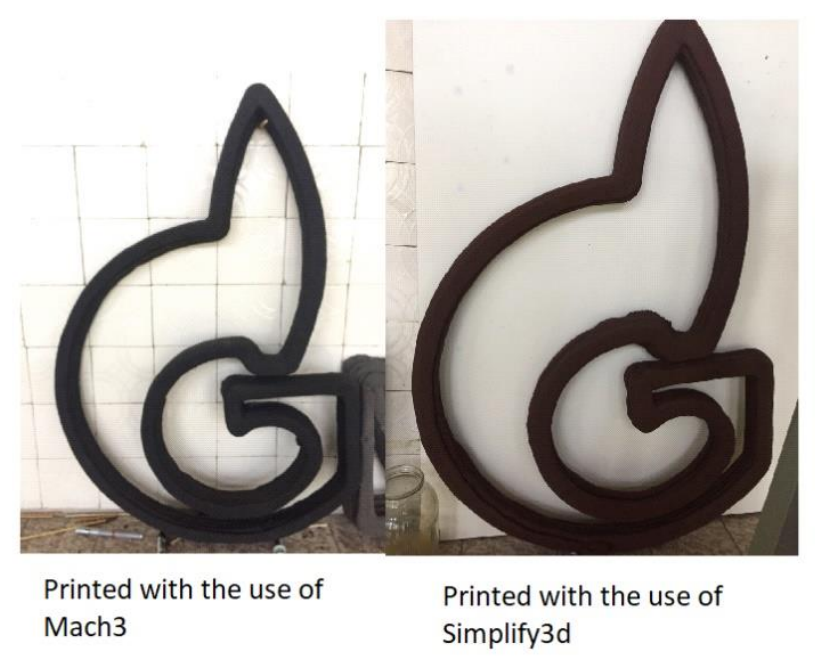

Fig. 4. Printing elements.

\section{Conclusion}

The construction printing can be used in any area with any composition. The main objective of the engineer consists in preparing the model conforming to requirements of technological process of the printing. In this research, two ways of the construction printing have been considered. First (AutoCad+SheetCam+Mach3) can be used for the printing of elements of typical and simple forms. It can be unique architectural elements as the chair, vase or 
elements of the house which do not have inclined parts. Second (SketchUp+Simplify3d) allows to prevent preparation of model for the printing. From the modelling program, the element is imported to Simplify3d, where work with the printer is performed directly. Thus, it is possible to print any form. Thus, the first way can be used for simple tasks, the second for enough difficult such as buildings with large number of apertures, and also inclined walls. This research work was supported by the Academic Excellence Project 5-100 proposed by Peter the Great St. Petersburg Polytechnic University.

\section{References}

1. V.N. Nerella, V. Mechtcherine, 3D Concrete Printing Technology, 333-347 (2019) https://doi.org/10.1016/b978-0-12-815481-6.00016-6

2. I. Su, G.S. Jung, N. Narayanan, M.J. Buehler, Curr. Opin. Biomed. Eng. (2020) https://doi.org/10.1016/j.cobme.2020.01.003

3. J.H. Lim, Y. Weng, Q.C. Pham, Constr. Build. Mater. 232 (2020) https://doi.org/10.1016/j.conbuildmat.2019.117075

4. J. Kruger, S. Zeranka, G. van Zijl, Autom. Constr. 106 (2019) https://doi.org/10.1016/j.autcon.2019.102904

5. B. Mazhoud, A. Perrot, V. Picandet, D. Rangeard, E. Courteille, Constr. Build. Mater. 214, 458-467 (2019) https://doi.org/10.1016/j.conbuildmat.2019.04.134

6. Q. Yuan, Z. Li, D. Zhou, T. Huang, H. Huang, D. Jiao, C. Shi, Constr. Build. Mater. 22, 7 (2019) https://doi.org/10.1016/j.conbuildmat.2019.07.326

7. J.G. Sanjayan, B. Nematollahi, 3D Concrete Printing Technology, 1-11 (2019) https://doi.org/10.1016/b978-0-12-815481-6.00001-4

8. R.J.M. Wolfs, F.P. Bos, T.A.M. Salet, Cem. Concr. Res. 119, 132-140 (2019) https://doi.org/10.1016/j.cemconres.2019.02.017

9. J. Katzer, T. Szatkiewicz, Constr. Build. Mater. 210, 157-161 (2019) https://doi.org/10.1016/j.conbuildmat.2019.03.204.

10. B. Furet, P. Poullain, S. Garnier, 3D printing for construction based on a complex wall of polymer-foam and concrete (2019) https://doi.org/10.1016/j.addma.2019.04.002

11. R.A. Buswell, W.R. Leal de Silva, S.Z. Jones, J. Dirrenberger, $3 D$ printing using concrete extrusion: A roadmap for research (2018) https://doi.org/10.1016/j.cemconres.2018.05.006

12. J. Xu, L. Ding, L. Cai, L. Zhang, H. Luo, W. Qin, Autom. Constr. 104, 95-106 (2019) https://doi.org/10.1016/j.autcon.2019.03.008

13. R. Duballet, O. Baverel, J. Dirrenberger, Autom. Constr. 83, 247-258 (2017) https://doi.org/10.1016/j.autcon.2017.08.018

14. V. Mechtcherine, V.N. Nerella, F. Will, M. Näther, J. Otto, M. Krause, Autom. Constr. 107 (2019) https://doi.org/10.1016/j.autcon.2019.102933

15. Y. Cui, D. Wang, J. Zhao, D. Li, S. Ng, Y. Rui, J. Build. Eng. 20, 21-29 (2018) https://doi.org/10.1016/j.jobe.2018.06.002

16. Z. Malaeb, F. AlSakka, F. Hamzeh, 3D Concrete Printing Technology, 115-136 (2019) https://doi.org/10.1016/b978-0-12-815481-6.00006-3

17. Y.W. Tay, Y. Qian, M.J. Tan, Compos. Part B Eng. 174 (2019) https://doi.org/10.1016/j.compositesb.2019.106968

18. Y. Zhang, Y. Zhang, W. She, L. Yang, G. Liu, Y. Yang, Constr. Build. Mater. 201, 278285 (2019) 
19. B. Panda, G.B. Singh, C. Unluer, M.J.Tan, J. Clean. Prod. 220, 610-619 (2019) https://doi.org/10.1016/j.jclepro.2019.02.185

20. Y. Zhang, Y. Zhang, G. Liu, Y. Yang, M. Wu, B. Pang, Constr. Build. Mater. 174, 263271 (2018) https://doi.org/10.1016/j.conbuildmat.2018.04.115

21. G. De Schutter, K. Lesage, V. Mechtcherine, V.N. Nerella, G. Habert, I. Agusti-Juan, Technical, economic and environmental potentials (2018) https://doi.org/10.1016/j.cemconres.2018.06.001 